\title{
Abundant and broad expression of transcription-induced chimeras and protein products in mammalian genomes
}

Guanting Lu ${ }^{1, @, *, ~ J i n ~ W u}{ }^{2,}{ }^{\circledR}$, Gangbin Zhao ${ }^{3}$, Zhiqiang Wang ${ }^{4}$ and Weihua Chen ${ }^{4}$

${ }^{1}$ Beijing DnaLead Science and Technology Co., LTD, Beijing, PR China;

${ }^{2}$ Department of Clinical Oncology, Xijing Hospital, the Fourth Military Medical University, Xi'an, China;

${ }^{3}$ Nanyang Institute of Technology, Henan, China;

${ }^{4}$ Key Laboratory of Genome Science and Information, Beijing Institute of Genomics, Chinese Academy of Sciences, Beijing, China.

${ }^{\circledR}$ These authors contributed equally to this work.

* To whom correspondence should be addressed: Beijing DnaLead Science and Technology Co., LTD, Beijing 102600, PR China. Email: Guantlv@ 126.com 


\section{ABSTRACT}

The expression of transcription-induced chimeras (TICs) was underestimated due to strategic and logical reasons. In order to thoroughly examine TICs, systematic survey of TIC events was conducted in mammalian genomes using ESTs, followed by experimental validation using RT-PCR and real-time quantitative PCR (qPCR). The expression of $\sim 98 \%$ TIC events in at least one tissue or cell line from both mouse and human was verified. Besides, $440 \%$ TICs were broadly expressed, and $\sim 33 \%$ of TICs showed expression levels comparable to or higher than their upstream parental genes. We further identified putative chimeric proteins in public databases and validated two using Western blotting. GO analysis revealed that proteins resided in one multiprotein complex or functioning in metabolic or signaling pathway tended to produce fused products. Taken together, we have shown substantial evidence for the underestimated TIC events; and TICs could be a novel regulated way to further increases the proteome complexity in mammalian genomes. Possible regulation mechanisms and evolution of TICs were also discussed.

KEYWORDS: TICS, EST, qPCR, genome

\section{INTRODUCTION}

It's now well established that the proteome complexity is far beyond the summation of protein-coding genes, due to alternative splicing, alternative transcription start sites (TSS) and transcription termination sites (TTSs) [1-8]. Recently, genome-wide analysis using expression sequence tags (ESTs) [9, 10], paired-end tags (PET) [11] 
and other resources [12-14] revealed an interesting phenomenon that may contribute to additional complexity of proteome in mammals: transcription-induced fusion of consecutive genes. In this scenario, a pair of tandem genes was co-transcribed, usually from the promoter of the upstream gene and to the end of the downstream gene; then the long primary transcript was spliced and merged into a chimeric mRNA containing exons from both genes. The two tandem genes were then called "parental genes". Typically, the transcribed intergenic regions were removed; but new exons could be derived if novel splicing sites were present. Although several cases of fusion RNAs were shown to be regulated and have unique expression patterns, such as HHLA1OC90 [15] and CD205-CD302 [16], some of them were restricted to cell lines and others considered as alternative spliced isoforms of known genes. Comparing to huge amount of EST sequences in public databases, only several hundreds of TIC events were identified and most of them were supported by only one EST sequence; thus TIC events were assumed random, non-regulated and of no biological significance.

Interestingly, a preliminary search in Refseq database at UCSC genome browser identified 66 and 21 Refgenes that were actually TICs fused by other Refgenes, in human and mouse, respectively. Majority of them and their parental genes were "reviewed" or "validated". These results had prompted us that TICs might be more abundant than expected, but somehow underestimated.

It's revealed that majority of the 66 human and 21 mouse TICs we identified in the Refseq database were not covered by previous studies $[9,10]$ and a public 
database, ChimeraDB [17]. In order to thoroughly examine the existence of TICs, a genome-wide survey was conducted in mammalian genomes using ESTs, followed by experimental validation using RT-PCR and qPCR. Western blotting experiments were also carried out to verify the protein products of selected TICs, since such evidence was only reported for a few fusion RNAs [18-20]. Finally we discussed possible molecular mechanisms that could contribute to TIC events and the evolution of TIC transcripts.

\section{MATERIALS AND METHODS}

\section{TIC events in public databases}

Datasets acquired from public databases were summarized in Table S6. Briefly, the human and mouse genome locations and the exact gene structures of Refseq genes were obtained from UCSC database. The Refseqs were then defined as "parental genes" for further searching of chimeric transcripts in public databases. Gene structures for other cDNA sequences were determined by aligning them to the genomes with BLAT [31]. The exact splicing positions in cDNAs were further corrected by SPIDEY [32]. GeneSplicer was then used to calculate the strength of each splicing site [33]. The sequence orientation was deduced from the splicing sites.

To eliminate artificial TICs that were derived from cDNA library construction, we used RESTRICT in the EMBOSS software suite [34] to scan restriction enzyme (RE) cutting sites in the joint cDNA sequences ( \pm 5 base pairs). 


\section{RT-PCR and qPCR}

RNAs from a variety of human and mouse tissues including liver, kidney, placenta, spleen, lung, and brain were purchased from Tianjin Saier Biotechnology. The cDNA was synthesized from $5 \mu \mathrm{g}$ of total RNA with M-MLV Reverse Transcriptase (Promega, US). The primers for reverse transcription PCR (PCR) and real-time quantitative PCR (qPCR) are listed in Table S7. The fragments of interest were sliced and cloned in the pGEM-Teasy vector (Promega) followed by sanger sequencing.

\section{RNA interference}

RNAi was performed against the parents genes of 3 co-transcripts, BCL2L2, CSNK2B and SLC43A3, respectively. The target region of RNAi and sequences of shRNA were listed in Table 4. The preparation of lentivirus particle was conducted according to the previous method [29]. After HeLa and K562 cells were at $85 \%$ confluency, $100 \mu \mathrm{L}$ crude lentivirus particles were added into the culture medium and co-cultured for $72 \mathrm{~h}$. Cell lysates were extracted for subsequent detection.

\section{Western blotting}

Proteins of human liver, muscle and kidney tissues were purchased from Tianjin Saier Biotechnology. The whole cell lysates were prepared with RIPA (radio immunoprecipitation assay) lysis buffer containing a protease inhibitor cocktail (Roche, Switzerland). The protein extracts were loaded onto 12\% SDS-PAGE gels followed by electrophoresis. After transferring to PVDF membrane (Millipore, USA), the proteins were immunoblotted with primary antibodies, including anti-BCL2L2 antibody (1:500, gift from Tianjin Saier Biotechnology), anti-CSNK2B antibody 
(1:200, Santa Cruz Biotechnology) and anti-PRG2 antibody (1:200, Santa Cruz Biotechnology). HRP-conjugated anti-rabbit and anti-mouse antibodies (1:1,000, Santa Cruz Biotechnology) were used as the secondary antibodies for chemiluminescence detection (GE Healthcare Life Sciences).

\section{RESULTS}

\section{Fusion Refseq genes in mouse and human}

66 and 21 entries in the Refseq database were identified as fusion transcripts of other Refgene genes for human and mouse, respectively. After examination of their Refgene status, we found that $63(95 \%)$ of human and $16(76 \%)$ of mouse fusion transcripts and both of their parental genes were marked as either "Reviwed" or "Validated". These TIC events were further grouped into 40 and 15 clusters according to their parental genes (Figure S1). Among them, $35(87.5 \%)$ and $12(80 \%)$ were not previously reported $[17,21,22]$ (Table S1).

\section{Fusion transcripts in public cDNA databases}

Additional 1,692 human and 508 mouse TIC transcripts were discovered in public cDNA databases (See Material and Methods) and grouped into 583 and 198 clusters, according to their parental genes in the two genomes. Among them, 361 and 158 clusters were not previously reported [17, 21, 22]. In total, 1,057 human and 372 mouse Refgenes were involved in TIC events, accounted for $5.7 \%$ and $2 \%$ of total genes in the two species (Table S1). 
Majority of the fusion events were supported by only one cDNA; less than $40 \%$ were supported by more than two ESTs (Figure S2A). The distributions of the fusion events on the two genomes were not random. In human, about 20 percent of the fusion events were located on chromosomes 1 and 19. In mouse, around 21 percent were found on chromosomes 2 and 7 (Figure S2B). We found significant correlation between the number of fusion events and the number of ESTs matched to chromosomes in human (Pearson correlation $=0.5402, \mathrm{p}=0.00642$ ), but not in mouse $(\operatorname{cor}=0.34, \mathrm{p}=0.13)$.

\section{Five major intergenic splicing patterns of co-transcripts}

As the RNA pol II complex goes through the intergenic region during cotranscription, we then examined the splicing patters of chimeric RNAs. As shown in Figure 1 and Table 3, five major intergenic splicing patterns were resolved according to the involvement of the exons of parental genes. Type 1 was the most common one which was seen in $28 \%$ of human and $34 \%$ of mouse co-transcripts. The common feature of type 2 and 3 was the exclusive usage of only one terminal exon in one parental gene and most exons in another one. Nearly $20 \%$ of human co-transcripts were in type 2 which kept the first exon of upper gene. In contrast, much less type 3 splicing, which included the last exon of lower gene, was found in our dataset $(7.5 \%$ in human).

Interestingly, in nearly $20 \%$ of human chimeric RNAs, a novel exon could be generated from the intergenic regions between two parental genes (Figure 1). 
Occasionally, more than one new exon was observed. Most of novel exons caused frame shift of the downstream gene. Finally, during the manual checking process, surprisingly we found 5 human and 1 mouse co-transcripts that involved 3 parental genes. We categorized these as the fifth type of intergenic splicing. Different splicing patterns were also observed in this type (Figure S3).

\section{Expression of fusion transcripts}

We chose 63 human and 20 mouse chimeric mRNAs and tested their expression in various tissues using RT-PCR. 62 (98.4\%) human and 19 (95\%) mouse chimeras were expressed in at least one tissue or cell lines; among them, we found that $26(41 \%)$ and $8(40 \%)$ were broadly expressed (in more than half of the tissues/ cell lines) in human and mouse, while the remaining were expressed in a tissue-specific manner (Table 1 and Table S2).

The expression abundance of 15 selected TIC clusters were tested by qPCR in multiple mouse tissues, and compared with parental genes and a housekeeping gene Polr $2 a$ (see methods). To our surprise, about five ( $33 \%)$ were expressed at similar or higher levels than the housekeeping control (Table 2).

\section{Fusion products in public databases}

It had been reported that although some fused transcripts appeared to have a comparable expression level with house-keeping genes, the in vivo existence of their correspondent proteins had not been identified so far [23]. In order to know whether 
chimeric proteins could be produced by the co-transcripts, we searched protein databases (SWISS-Prot and NCBI) for the presence of proteins matching the identified co-transcripts by BLASTX, followed by manual inspection. Totally, 105 and 12 chimeric proteins were identified in human and mouse, respectively (Table S3).

\section{In vivo identification of fusion proteins by Western blotting}

We tested the in vivo existence of protein products of three human TICs in HeLa, fetal liver, muscle and K-562 lysates. The corresponding genomic regions of the TICs were amplified in the HeLa cells and sequenced to rule out the influence of genomic rearrangement. The corresponding protein produced by BCL2L2-PABPN1 was detected in HeLa whole lysates. As the BCL2L2 was down-regulated by RNAi, the corresponding chimeric protein disappeared. The chimeric protein of CSNK2BLY6G5B was identified in K-562 whole lysate by monoclonal anti-CSNK2B (Nterminal domain) but not in muscle. After CSNK2B was suppressed by RNAi, the expression of parental and chimeric proteins were reduced. The detection of SLC43A3-PRG2 wasn't detected by the monoclonal anti-PRG2 (C-terminal domain) in HeLa, liver and spleen (Figure 2).

\section{Evolution of fusion transcripts in vertebrates}

The above observations of co-transcription in human and mouse genomes raised a question if co-transcription resulted from "fusion" of two tandem genes or split of one larger gene. In order to know to what extent the co-transcripts conserved in other organisms, we compared the human chimeric transcripts with ESTs and mRNAs in 
the available databases of chimpanzee, mouse, rat, pig, horse, chicken and Xenopus laevis. In total we found 80 co-transcripts that existed in at least one of the organisms mentioned above (Table S4). More than 50\% were conserved in mouse.

More interestingly, corresponding to human co-transcripts, we indeed discovered 31 human co-transcripts which were actually single large genes in other organisms (Figure 3 and Figure S4, Table S4). Among them, 3 overlapped with the abovementioned 80 conserved co-transcripts as these messengers were shown to be transcribed from two tandem genes in one animal but to be the product of a single gene in other organism. Further analysis demonstrated that most of the ancestral large genes were found in mouse, rat and Bos tarus (Figure 3). This suggested that in human, certain chimeric RNAs might be occurred by gene splitting mechanism as to keep the ancestral gene function simultaneously with two new transcripts produced.

\section{Functionally- or physically-related genes tend to be co-transcribed}

To find out what genes tend to be co-transcribed, we performed GO analysis to the parental genes of the 80 conserved co-transcripts and those of the 31 co-transcripts having single gene in other species.

As for the conserved co-transcripts with both of their parents having GO items, majority of them (96.3\%) were produced by the fusion of exons of two parental genes which didn't have single ancestral genes in other organisms. About two third of these fused transcripts in this category fell into type I intergenic splicing. In the course of evolution, the fused mRNAs could be fixed as new single genes by combining exons 
of their parental genes. GO analysis revealed that proteins localizing in one multiprotein complexes or functioning in one metabolic or signal transduction pathway tend to produce fused products (Table S3).

For the 31 co-transcripts having single ancestral genes in other organisms, we found that about $96 \%$ of the parental genes are physically or functionally interrelated. The proteins were components in signal transduction pathways, transcription factors complexes, enzymes involved in metabolic pathways, electron transfer chain proteins and other molecular processes (Table S3). This indicated that during evolution, the single ancestral gene gained new regulatory regions in the middle and then split into separated daughter genes containing exons from their mother.

\section{DISCUSSIONS}

In the present study, our primary goal was to prove that fusion transcripts could contribute additional complexity to mammalian proteome. Chimeric transcripts had been reported decades ago [24]. However, comparing to huge amount of EST sequences in public databases, the number of TIC events was relative very small. Surprisingly, during a preliminary search of Refseq genes of mouse and human, we identified about 100 fusion events where one Refseq was actually fusion transcript from two other Refseq genes; most of human (90\%) and majority of mouse (61\%) fusion transcripts and their parental genes were marked as either "Reviewed" or "Validated", raised the possibility that the fusion events were regulated and likely functional. 
Comparing with previous studies [9, 10] and the ChimericDB database [17], about $90 \%$ human and $80 \%$ mouse fusion Refgene events were novel. Additional 1,692 human and 508 mouse fusion transcripts were identified in public database, and further grouped into 583 and 198 clusters according to their parental genes in human and mouse, respectively. Compared with previous co-transcript studies, 361 (61.9\%) and $158(80 \%)$ of novel fusion cases were identified in human and mouse, respectively. We plotted all results in a similar manner with the UCSC Genome Browser (Figure S1) and checked manually. In total, about $5.7 \%$ and $2 \%$ Refgenes in human and mouse were involved in fusion transcripts, which is much higher than previously observed but very close to the authors' estimation $[9,10]$.

After analyzing the intergenic splicing pattern, the co-transcription events could be categorized into 5 major splicing patterns. Type 1 seemed to detour the translation stop codon in the 3'-UTR of the upstream genes to generate functional fused transcripts than to accumulate mutations at the stop codon during evolution [25]. Novel genes produced by gene fusion had been described in lower organisms [26, 27]. The type II intergenic splicing produced new first exons for the downstream genes. Because the first exon often contained regulatory motifs which controlled gene expression, this type of co-transcription-induced first exon (CoTIFE) could put the expression of downstream gene under the control of upstream gene's regulatory motifs transcriptionally (by promoters) and translationally (by 5'-UTR). Together with the first exon of the downstream gene itself, CoTIFE could further fine-tune the 
expression of downstream genes in the same way as the upstream genes or change the patterns of tissue-specific expression of the downstream genes [7, 27]. In addition to regulate the expression of downstream gene, CoTIFE could add new N-terminal to the proteins translated by the co-transcripts [7, 28]. On contrary to type II intergenic splicing, type III rendered the upstream gene containing the terminal exons of the downstream genes. Since terminal exons often contained motifs responsible for the efficacy of transcription termination and stability of mRNAs, these co-transcriptioninduced terminal exons (CoTITEs) could play an important role in regulating the expression of upstream parental genes of co-transcripts. This led us to have a deeper understanding of the complexity of gene regulation in eukaryotes.

Consistent with previous findings, the intergenic distance of the parental genes in fusion events was significantly shorter $\left(P<10^{-15}\right.$, Wilcoxon Rank Sum test $)$ than that of all tandem neighboring gene pairs in human and mouse, respectively (Figure S2, C-D). However, among 4,100 human and 4,900 mouse tandem gene pairs, only $363(8.8 \%)$ and $120(2.4 \%)$ produced chimeric mRNAs, suggesting intergenic distance may not be the determinant contributor to fusion events.

During co-transcription of parental genes, it appears the RNA pol II machinery does not stop at the termination sites of upstream genes. Therefore we wished to know if the ploy(A) sites in intergenic regions and the 5' splicing of the last exon in upstream gene were weaker and consequently led to read through by pol II. However, our analysis indicated that the fusion transcripts did not contain weaker poly(A) 
signals $(P>0.5)$ (Figure S2, E-F). From this analysis, it appears that these mechanical features including intergenic distances, strengths of poly(A) signals and 5' splicing sites of the last exon in the upstream gene were not major contributors to fusion transcripts.

The number of fusion transcripts was much lower comparing to huge amount of mammalian EST sequences. One major reason is that only ESTs span the joint regions will be counted. Furthermore, most EST analysis pipelines exclude so-called "chimeric" clones from further analysis [26-29]. Therefore we validated the expression of fusion transcripts in multiple human and mouse tissues using RT-PCR. The idea was not only to verify the existence of the transcripts, as was previously tested [21, 22], but also test the expression variations in different tissues. We selected randomly fusion transcripts and validated more than $95 \%$ for human and mouse. Surprisingly, among the validated transcripts, around $40 \%$ were ubiquitously expressed. Furthermore, qPCR analysis indicated that more than one third of the fusion transcripts in both genomes expressed in similar or more abundant to upstream parental genes and/or housekeeping gene, Polr2a. Therefore, we provided evidences for broad and abundant expression of fusion transcripts in mammalian genomes.

We also provided evidences of protein products for fusion transcripts by searching public protein databases and in vivo validation using Western blotting. In total, we found 105 and 12 fusion proteins for human and mouse, respectively; among 
which, STON1-GTF2A1L [20], NME1-NME2 [30] and TMEM189-UBE2V1 [19] were also confirmed by RT-PCR.

A portion of chimeric gene pairs in human appeared to exist as both two individual genes and a large fused gene; most of the fused genes encode larger proteins containing multi-domains. Considering the frequent production of fused mRNAs, co-transcriptive genes may represent a new type of transcription units. In addition to the "classical" transcription units, these co-transcriptional units are composed of two or more adjacent genes.

\section{ACKNOWLEDGEMENTS}

This work was supported by funding from the National Science Foundation of China (81101490 to GL).

\section{REFERENCES}

1. Maniatis, T.,B. Tasic. Alternative pre-mRNA splicing and proteome expansion in metazoans. Nature, 2002, 418: 236-43

2. Xing, Y.,C. Lee. Relating alternative splicing to proteome complexity and genome evolution. Adv Exp Med Biol, 2007, 623: 36-49

3. Zavolan, M., S. Kondo, C. Schonbach, J. Adachi, D.A. Hume. Impact of alternative initiation, splicing, and termination on the diversity of the mRNA transcripts encoded by the mouse transcriptome. Genome Res, 2003, 13: 1290-300

4. Huby, T., C. Dachet, R.M. Lawn, J. Wickings, M.J. Chapman. Functional analysis of the chimpanzee and human apo(a) promoter sequences: identification of sequence variations responsible for elevated transcriptional activity in chimpanzee. J Biol Chem, 2001, 276: 22209-14 
5. Carninci, P., A. Sandelin, B. Lenhard, S. Katayama, K. Shimokawa. Genomewide analysis of mammalian promoter architecture and evolution. Nat Genet, 2006, 38: 626-35

6. Wang, B.B.,V. Brendel. Genomewide comparative analysis of alternative splicing in plants. Proc Natl Acad Sci U S A, 2006, 103: 7175-80

7. Chen, W.H., G. Lv, C. Lv, C. Zeng, S. Hu. Systematic analysis of alternative first exons in plant genomes. BMC Plant Biol, 2007, 7: 55

8. Kimura, K., A. Wakamatsu, Y. Suzuki, T. Ota, T. Nishikawa. Diversification of transcriptional modulation: large-scale identification and characterization of putative alternative promoters of human genes. Genome Res, 2006, 16: 55-65

9. Akiva, P. Transcription-mediated gene fusion in the human genome. Genome Res, 2005, 16: 30-36

10. Parra, G. Tandem chimerism as a means to increase protein complexity in the human genome. Genome Res, 2005, 16: 37-44

11. Ruan, Y., H.S. Ooi, S.W. Choo, K.P. Chiu, X.D. Zhao. Fusion transcripts and transcribed retrotransposed loci discovered through comprehensive transcriptome analysis using Paired-End diTags (PETs). Genome Res, 2007, 17: 828-38

12. Djebali, S., P. Kapranov, S. Foissac, J. Lagarde, A. Reymond. Efficient targeted transcript discovery via array-based normalization of RACE libraries. Nat Methods, 2008, 5: 629-35

13. Birney, E., J.A. Stamatoyannopoulos, A. Dutta, R. Guigo, T.R. Gingeras. Identification and analysis of functional elements in $1 \%$ of the human genome by the ENCODE pilot project. Nature, 2007, 447: 799-816

14. Denoeud, F., P. Kapranov, C. Ucla, A. Frankish, R. Castelo. Prominent use of distal 5' transcription start sites and discovery of a large number of additional exons in ENCODE regions. Genome Res, 2007, 17: 746-59

15. Kowalski, P.E., J.D. Freeman, D.L. Mager. Intergenic splicing between a HERV-H endogenous retrovirus and two adjacent human genes. Genomics, 1999, 57: 371-9

16. Kato, M., S. Khan, N. Gonzalez, B.P. O'Neill, K.J. McDonald. Hodgkin's lymphoma cell lines express a fusion protein encoded by intergenically spliced mRNA for the multilectin receptor DEC-205 (CD205) and a novel C-type lectin receptor DCL-1. J Biol Chem, 2003, 278: 34035-41

17. Kim, N., P. Kim, S. Nam, S. Shin, S. Lee. ChimerDB--a knowledgebase for fusion sequences. Nucleic Acids Res, 2006, 34: D21-4

18. Pradet-Balade, B., J.P. Medema, M. Lopez-Fraga, J.C. Lozano, G.M. Kolfschoten. An endogenous hybrid mRNA encodes TWE-PRIL, a functional cell surface TWEAK-APRIL fusion protein. EMBO J, 2002, 21: 5711-20

19. Thomson, T.M., J.J. Lozano, N. Loukili, R. Carrio, F. Serras. Fusion of the human gene for the polyubiquitination coeffector UEV1 with Kua, a newly identified gene. Genome Res, 2000, 10: 1743-56 
20. Upadhyaya, A.B., S.H. Lee, J. DeJong. Identification of a general transcription factor TFIIAalpha/beta homolog selectively expressed in testis. J Biol Chem, 1999, 274: 18040-8

21. Akiva, P., A. Toporik, S. Edelheit, Y. Peretz, A. Diber. Transcriptionmediated gene fusion in the human genome. Genome Res, 2006, 16: 30-6

22. Parra, G., A. Reymond, N. Dabbouseh, E.T. Dermitzakis, R. Castelo. Tandem chimerism as a means to increase protein complexity in the human genome. Genome Res, 2006, 16: 37-44

23. Roux, M., H. Leveziel, V. Amarger. Cotranscription and intergenic splicing of the PPARG and TSEN2 genes in cattle. BMC Genomics, 2006, 7: 71

24. Fears, S., C. Mathieu, N. Zeleznik-Le, S. Huang, J.D. Rowley. Intergenic splicing of MDS1 and EVI1 occurs in normal tissues as well as in myeloid leukemia and produces a new member of the PR domain family. Proc Natl Acad Sci U S A, 1996, 93: 1642-7

25. Long, M. A new function evolved from gene fusion. Genome Res, 2000, 10: $1655-7$

26. Marcotte, E.M., M. Pellegrini, H.L. Ng, D.W. Rice, T.O. Yeates. Detecting protein function and protein-protein interactions from genome sequences. Science, 1999, 285: 751-3

27. Kitagawa, N., T. Washio, S. Kosugi, T. Yamashita, K. Higashi. Computational analysis suggests that alternative first exons are involved in tissue-specific transcription in rice (Oryza sativa). Bioinformatics, 2005, 21: 1758-63

28. Zent, C.S., C. Mathieu, D.F. Claxton, D.E. Zhang, D.G. Tenen. The chimeric genes AML1/MDS1 and AML1/EAP inhibit AML1B activation at the CSF1R promoter, but only AML1/MDS1 has tumor-promoter properties. Proc Natl Acad Sci U S A, 1996, 93: 1044-8

29. Shin, K.J., E.A. Wall, J.R. Zavzavadjian, L.A. Santat, J. Liu. A single lentiviral vector platform for microRNA-based conditional RNA interference and coordinated transgene expression. Proc Natl Acad Sci U S A, 2006, 103: 13759-64

30. Valentijn, L.J., J. Koster, R. Versteeg. Read-through transcript from NM23$\mathrm{H} 1$ into the neighboring NM23-H2 gene encodes a novel protein, NM23-LV. Genomics, 2006, 87: 483-9

31. Kent, W.J. BLAT--the BLAST-like alignment tool. Genome Res, 2002, 12 : 656-64

32. Wheelan, S.J., D.M. Church, J.M. Ostell. Spidey: a tool for mRNA-togenomic alignments. Genome Res, 2001, 11: 1952-7

33. Pertea, M., X. Lin, S.L. Salzberg. GeneSplicer: a new computational method for splice site prediction. Nucleic Acids Res, 2001, 29: 1185-90

34. Rice, P., I. Longden, A. Bleasby. EMBOSS: the European Molecular Biology Open Software Suite. Trends Genet, 2000, 16: 276-7 


\section{FIGURE LEGENDS}

Figure 1. Diagram of the five major intergenic splicing patterns of co-transcripts

Type I represented for co-transcripts which removed the terminal exon of upstream gene and first exon of the downstream gene. Type II happened between the very first exon of upstream gene and any exons of the downstream gene. Type III was just contrary to type II co-transcripts which involved any exons of upstream gene and only the terminal exon of the downstream gene. Type IV co-transcripts contained novel exons produced by intergenic region. Type $\mathrm{V}$ fused mRNAs were generated by 3 parental genes. The arrow represents direction of transcription. The solid lines stood for definite splicing patterns; dashed lines represents possible splicing patterns.

\section{Figure 2. Western blotting for the selected TICs}

We chose to validate the protein products of two TIC mRNAs, BCL2L2-PABPNI and $C S N K 2 B-L Y 6 G 5 B$. Genomic positions of the fusion transcriptions and their parental genes are shown in (A) and (D); boxes represent exons, with thinner boxes representing the untranslated regions (UTRs); the thin lines and arrows donate introns and transcription orientation, respectively. RT-PCR results in different human tissues are shown in (B) and (E). Results of Western blotting experiments are shown in (C) and $(\mathrm{F})$.

Figure 3. Conservation of a human fusion transcript and parental genes in mouse 
In human genome, CREM (NM_183060) is a fusion transcript by CREM isoform b (NM_001881) and CREM isoform f (NM_182719). All three have Refgene status of "reviewed" or "validated", according to the UCSC genome browser. Orthologous partners for all three transcripts are found in mouse. Poly(A) signals based on mapped ESTs (purple) and SVM predictions (green) for human genomes are shown.

Figure 4. Recent splitting events in human and mouse due to newly derived CpG islands

A fusion transcript of $U N K L$ and C16orf 28 was detected in human (C). Orthologous genes of this fusion transcript and $U N K L$ could be found in mouse (B). However, in $X$. tropicalis, only the fusion transcript existed (A). 


\section{Tables}

\section{Table 1}

Table 1. Expression of verified human TICs by RT-PCR (only selected genes shown here; a full list is available at supplementary Table S1)

\begin{tabular}{|c|c|c|c|c|c|c|c|c|c|c|c|c|c|}
\hline \multirow{2}{*}{$\begin{array}{l}\text { TIC } \\
\text { (Upstream/Downstream } \\
\text { parents) }\end{array}$} & \multicolumn{8}{|c|}{ Cell lines } & \multicolumn{5}{|c|}{ Fetal tissues } \\
\hline & BJAB & Raji & A549 & HeLa & $\begin{array}{l}\text { SGC- } \\
7901\end{array}$ & GLC82 & LX-2 & HEK293 & Liver & Kidney & Spleen & Lung & Brain \\
\hline INS/IGF2 & $\sqrt{ }$ & $\sqrt{ }$ & & & $\sqrt{ }$ & & $\sqrt{ }$ & & $\sqrt{ }$ & $\sqrt{ }$ & & & \\
\hline SCP2/SCP2 ${ }^{*}$ & $\sqrt{ }$ & $\sqrt{ }$ & $\sqrt{ }$ & & & $\sqrt{ }$ & $\sqrt{ }$ & & $\sqrt{ }$ & $\sqrt{ }$ & $\sqrt{ }$ & $\sqrt{ }$ & $\sqrt{ }$ \\
\hline PMF1/BGLAP & $\sqrt{ }$ & $\sqrt{ }$ & & $\sqrt{ }$ & & $\sqrt{ }$ & $\sqrt{ }$ & $\sqrt{ }$ & $\sqrt{ }$ & $\sqrt{ }$ & & & $\sqrt{ }$ \\
\hline NMW1/NME2 * & $\sqrt{ }$ & $\sqrt{ }$ & $\sqrt{ }$ & & & $\sqrt{ }$ & $\sqrt{ }$ & $\sqrt{ }$ & $\sqrt{ }$ & $\sqrt{ }$ & $\sqrt{ }$ & $\sqrt{ }$ & $\sqrt{ }$ \\
\hline RBM14/RBM4 * & $\sqrt{ }$ & $\sqrt{ }$ & $\sqrt{ }$ & $\sqrt{ }$ & $\sqrt{ }$ & $\sqrt{ }$ & $\sqrt{ }$ & $\sqrt{ }$ & $\sqrt{ }$ & $\sqrt{ }$ & $\sqrt{ }$ & $\sqrt{ }$ & $\sqrt{ }$ \\
\hline CCL15/CCL14 & & & & & & & & & $\sqrt{ }$ & & & & \\
\hline C20orf35/BNDD2 * & $\sqrt{ }$ & $\sqrt{ }$ & $\sqrt{ }$ & $\sqrt{ }$ & $\sqrt{ }$ & $\sqrt{ }$ & $\sqrt{ }$ & & $\sqrt{ }$ & $\sqrt{ }$ & $\sqrt{ }$ & $\sqrt{ }$ & $\sqrt{ }$ \\
\hline BLOC1S1/RDH5 & $\sqrt{ }$ & $\sqrt{ }$ & $\sqrt{ }$ & $\sqrt{ }$ & $\sqrt{ }$ & $\sqrt{ }$ & $\sqrt{ }$ & $\sqrt{ }$ & $\sqrt{ }$ & $\sqrt{ }$ & $\sqrt{ }$ & $\sqrt{ }$ & $\sqrt{ }$ \\
\hline STON1/GTF2A1L & $\sqrt{ }$ & $\sqrt{ }$ & $\sqrt{ }$ & & $\sqrt{ }$ & $\sqrt{ }$ & $\sqrt{ }$ & $\sqrt{ }$ & $\sqrt{ }$ & $\sqrt{ }$ & $\sqrt{ }$ & $\sqrt{ }$ & \\
\hline ANKRD39/ANKRD23 * & $\sqrt{ }$ & $\sqrt{ }$ & $\sqrt{ }$ & $\sqrt{ }$ & $\sqrt{ }$ & $\sqrt{ }$ & $\sqrt{ }$ & & & & $\sqrt{ }$ & $\sqrt{ }$ & \\
\hline APITD1/CORT $^{*}$ & $\sqrt{ }$ & $\sqrt{ }$ & $\sqrt{ }$ & & & & $\sqrt{ }$ & & & $\sqrt{ }$ & $\sqrt{ }$ & $\sqrt{ }$ & \\
\hline HOXC6/HOXC5 & $\sqrt{ }$ & & & & & & & & & & & & \\
\hline MUTED/TXNDC5 & $\sqrt{ }$ & $\sqrt{ }$ & & & & & & & & & $\sqrt{ }$ & & \\
\hline SYNJ2BP/C14orf112 * & $\sqrt{ }$ & & $\sqrt{ }$ & $\sqrt{ }$ & $\sqrt{ }$ & $\sqrt{ }$ & $\sqrt{ }$ & $\sqrt{ }$ & $\sqrt{ }$ & $\sqrt{ }$ & $\sqrt{ }$ & $\sqrt{ }$ & \\
\hline RPS10/NUDT3 & $\sqrt{ }$ & $\sqrt{ }$ & & $\sqrt{ }$ & & & & $\sqrt{ }$ & & & & $\sqrt{ }$ & \\
\hline C1QTNF6/IL2RB & & $\sqrt{ }$ & & $\sqrt{ }$ & & & $\sqrt{ }$ & & & & $\sqrt{ }$ & & \\
\hline PNKD/PNKD & & $\sqrt{ }$ & $\sqrt{ }$ & & $\sqrt{ }$ & & & & & & & $\sqrt{ }$ & $\sqrt{ }$ \\
\hline NUP62/IL4I1 & $\sqrt{ }$ & $\sqrt{ }$ & & & & & & & $\sqrt{ }$ & & $\sqrt{ }$ & & $\sqrt{ }$ \\
\hline
\end{tabular}




\begin{tabular}{|l|l|l|l|l|l|l|l|l|l|l|l|l|l|}
\hline Kua/UBE2V1 * & * & $\sqrt{ }$ & $\sqrt{ }$ & & & $\sqrt{ }$ & & & & & $\sqrt{ }$ & $\sqrt{ }$ & $\sqrt{ }$ \\
\hline
\end{tabular}

"Genes that were broadly expressed.

\section{Table2}

Table 2. Expression of selected mouse TICs by quantitative PCR.

\begin{tabular}{|c|c|c|c|c|c|c|c|c|c|c|c|c|c|c|c|}
\hline & \multicolumn{4}{|l|}{ Liver } & \multicolumn{4}{|l|}{ Lung } & \multicolumn{4}{|c|}{ Muscle } & \multicolumn{3}{|c|}{ Spleen } \\
\hline & TIC & $\begin{array}{l}\text { upstream } \\
\text { parent }\end{array}$ & $\begin{array}{l}\text { downstream } \\
\text { parent }\end{array}$ & Polr2a & TIC & $\begin{array}{l}\text { upstream } \\
\text { parent }\end{array}$ & $\begin{array}{l}\text { downstream } \\
\text { parent }\end{array}$ & Polr2a & TIC & $\begin{array}{l}\text { upstream } \\
\text { parent }\end{array}$ & $\begin{array}{l}\text { downstream } \\
\text { parent }\end{array}$ & Polr2a & TIC & $\begin{array}{l}\text { upstream } \\
\text { parent }\end{array}$ & $\begin{array}{l}\text { downst } \\
\text { parent }\end{array}$ \\
\hline Doc4/Apoc2 & 26.14 & 14.95 & 17.02 & 19.29 & & & & & & & & & & & \\
\hline $\mathrm{rc} / \mathrm{Cela2a}{ }^{*}$ & & & & & 26.37 & 26.07 & 27.12 & 14.81 & & & & & & & \\
\hline $\mathrm{dx} 17 / \mathrm{Ddx} 17$ & 23.66 & 23.96 & 23.11 & 19.29 & 15.74 & 15.09 & 14.89 & 14.81 & 19.60 & 17.86 & 17.52 & 18.52 & 19.17 & 16.77 & 18.02 \\
\hline e2/Ece2 & 29.94 & 22.86 & 21.89 & 19.29 & & & & & & & & & & & \\
\hline i2a/Evi2b & 28.01 & 24.06 & 22.17 & 19.29 & 22.13 & 19.32 & 21.42 & 14.81 & & & & & & & \\
\hline t15/Cluap1 ${ }^{*}$ & & & & & 21.96 & 21.01 & 22.75 & 14.81 & & & & & & & \\
\hline up62/II4i1 & 28.22 & 23.10 & 30.19 & 19.29 & & & & & & & & & & & \\
\hline kd/Pnkd & 27.55 & 22.02 & 23.30 & 19.29 & 22.97 & 19.32 & 21.45 & 14.81 & 23.64 & 19.25 & 20.61 & 18.52 & & & \\
\hline amp2/Vps25 & 30.79 & 27.23 & 31.02 & 19.29 & & & & & 24.58 & 19.45 & 23.22 & 18.52 & 24.12 & 17.91 & 20.32 \\
\hline om14/Rbm4 & & & & & 19.17 & 17.09 & 17.59 & 14.81 & 22.22 & 20.24 & 18.52 & 18.52 & & & \\
\hline Imo3/Ube2g2 & & & & & & & & & 25.34 & 19.21 & 22.23 & 18.52 & & & \\
\hline /nj2bp/Cox16 & 25.89 & 20.60 & 25.04 & 19.29 & 21.84 & 18.34 & 20.27 & 14.81 & 20.78 & 17.57 & 19.92 & 18.52 & 23.02 & 19.60 & 20.20 \\
\hline Ifsf12/Tnfsf13 & 26.21 & 30.12 & 28.43 & 19.29 & 21.85 & 28.20 & 25.85 & 14.81 & 23.11 & 29.10 & 27.26 & 18.52 & 22.40 & 26.91 & 24.27 \\
\hline p566/Zfp82 * & 25.31 & 25.98 & 26.03 & 19.29 & 21.34 & 21.99 & 20.59 & 14.81 & 22.64 & 23.17 & 23.83 & 18.52 & & & \\
\hline xx1/9130401M01Rik & & & & & & & & & 21.97 & 20.17 & NA & 18.52 & & & \\
\hline
\end{tabular}

"TICs with expression abundances higher than or similar to parental genes or housekeeping control (Polr2a). 
Ct values from realtime quantitative PCR experiments are shown here. Higher values indicate lower expression, lower values indicate higher expression. NA, detection failed.

Table 3.

Table 3 Amount of the five types of co-transcripts in human and mouse.

\begin{tabular}{lll}
\hline Types & Human & Mouse \\
\hline Type I & 163 & 68 \\
Type II & 113 & 36 \\
Type III & 44 & 13 \\
Type IV & 115 & 25 \\
Type V & 5 & 1 \\
\hline
\end{tabular}


Table 4

Table 4 RNAi target region and shRNA squence

\begin{tabular}{|l|l|l|l|}
\hline Genes & No. & Target region of RNAi & shRNA sequence* $^{*}$ \\
\hline BCL2L2 & RNAi_1 & TGGCAGACTTTGTAGGTTATA & CCGG-TGGCAGACTTTGTAGGTTATA-CTCGAG-TATAACCTACAAAGTCTGCCA-TTTTTG \\
\hline & RNAi_2 & CAGAAGGGTTATGTCTGTGGA & CCGG-CAGAAGGGTTATGTCTGTGGA-CTCGAG-TCCACAGACATAACCCTTCTG-TTTTTG \\
\hline CSNK2B & RNAi_1 & TGGGCTCCGTGGCAATGAATT & CCGG-TGGGCTCCGTGGCAATGAATT-CTCGAG-AATTCATTGCCACGGAGCCCA-TTTTTG \\
\hline SLC43A3 & RNAi_1 & CCTCGGCAGTCTTCCTTATTA & CCGG-CCTCGGCAGTCTTCCTTATTA-CTCGAG-TAATAAGGAAGACTGCCGAGG-TTTTTG \\
\hline
\end{tabular}

*bold character: target region of RNAi in the shRNA. 


\section{Figures}

figure 1 five major pattern of splicing
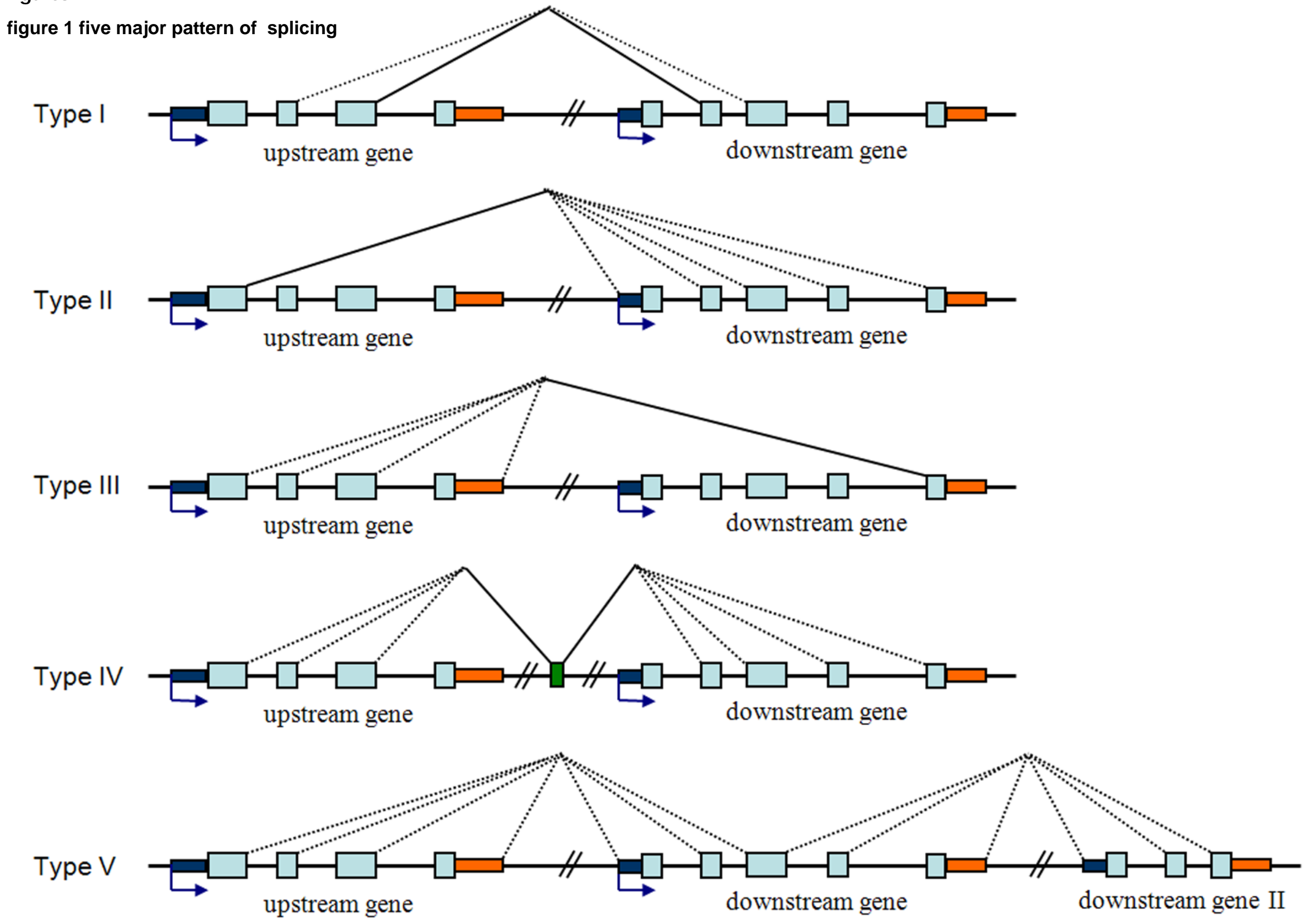
figure 2. western blotting experiments for selected tic

A

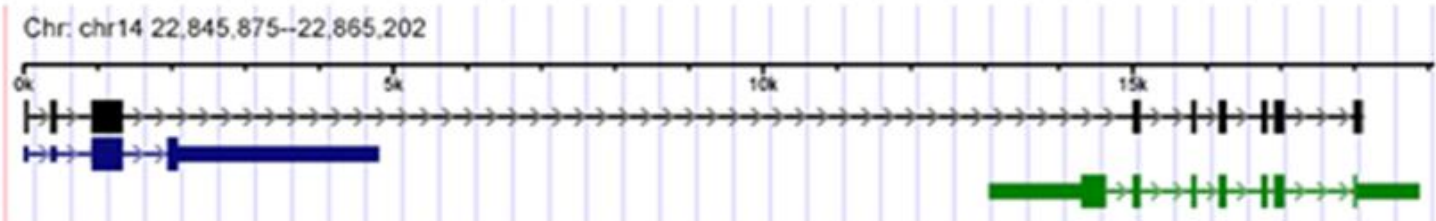

B

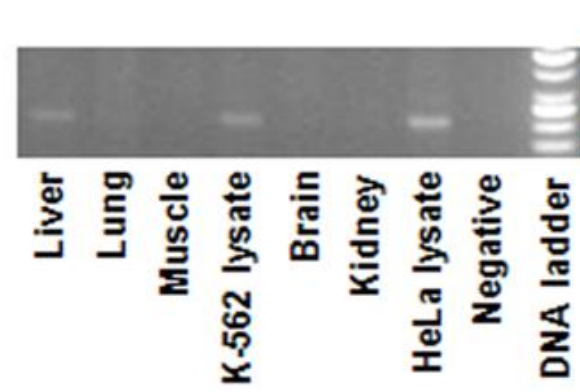

C

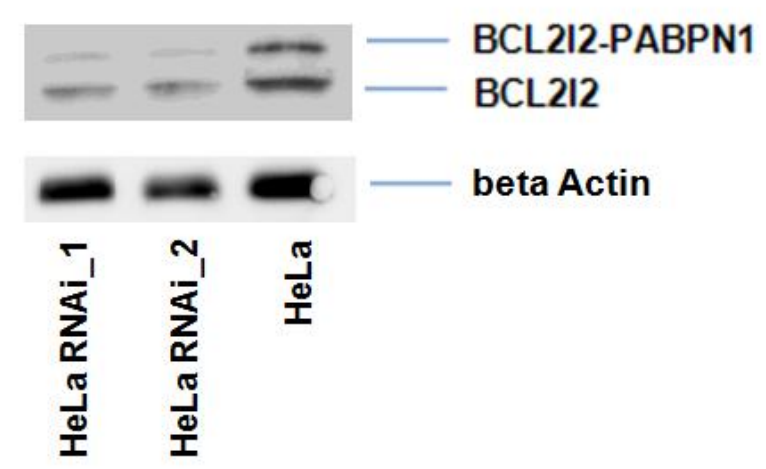

D

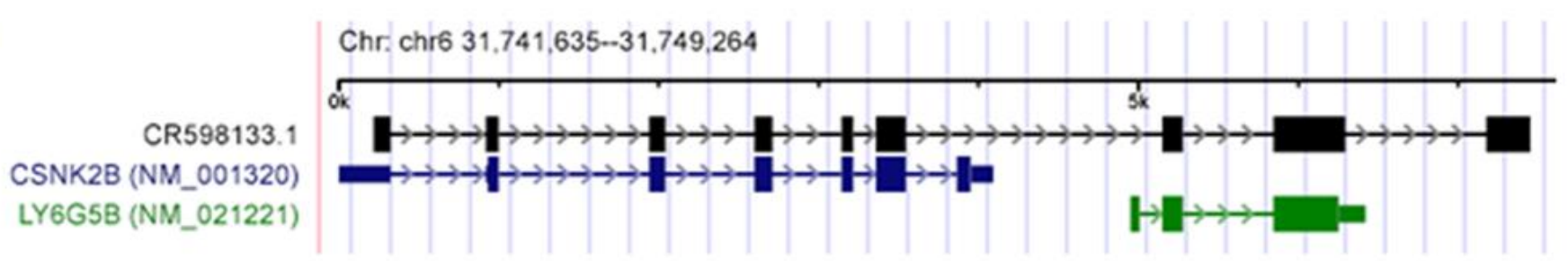

E
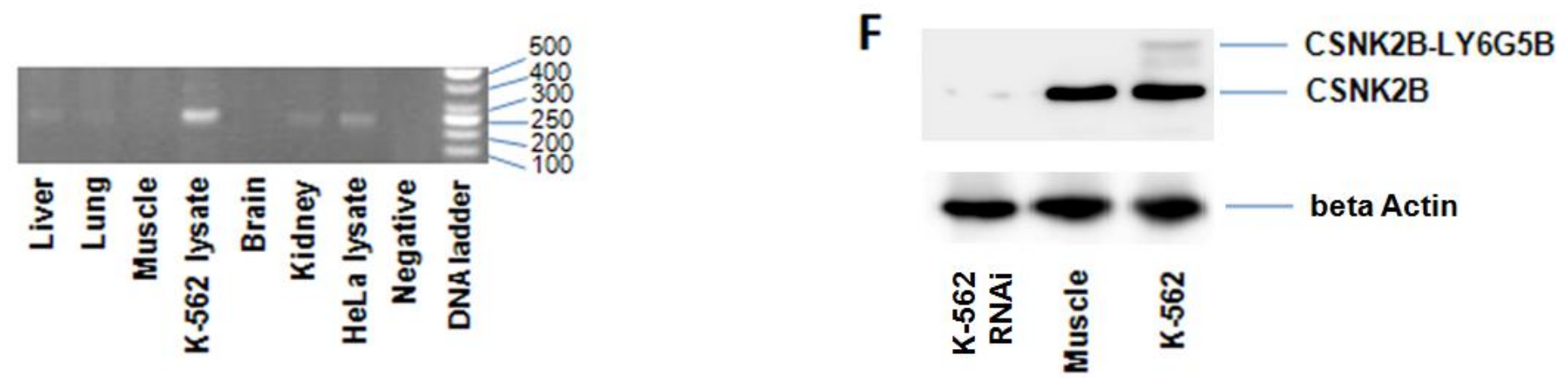

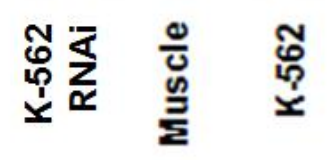

Figure 3. western blotting experiments for selected TIC 
figure 3. example of conserved tic and parental genes

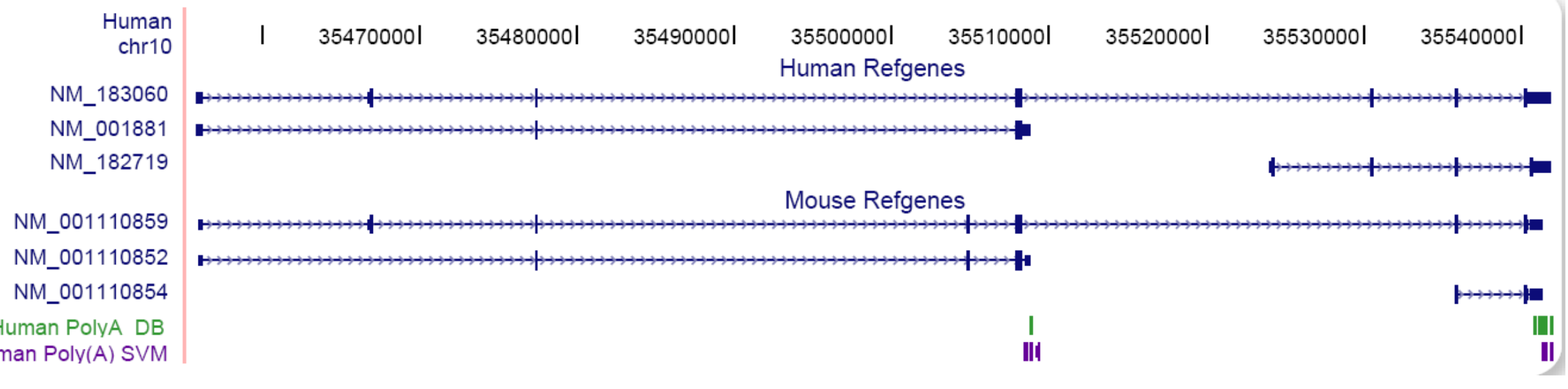

Figure 4. Example of conserved TIC and parental genes

\section{figure 3.}




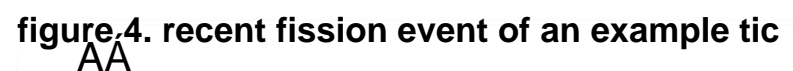

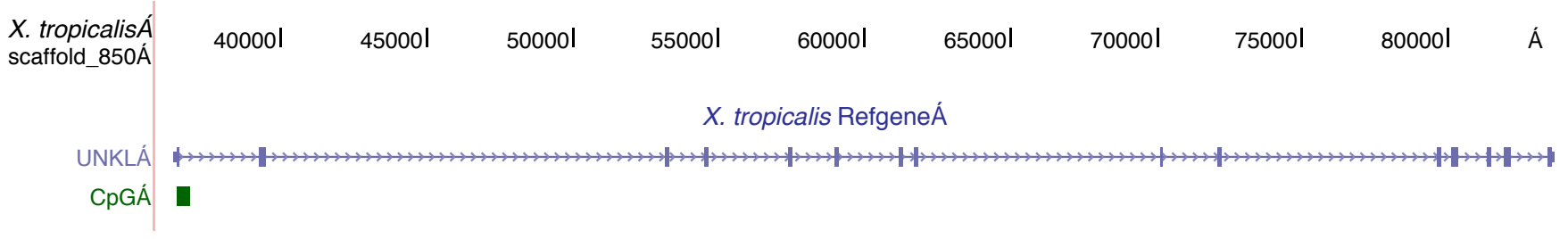

B

\begin{tabular}{|c|c|c|c|c|c|c|c|c|c|}
\hline $\begin{array}{r}\text { Mouse } \\
\text { chr17 }\end{array}$ & $25330000^{1}$ & $25335000^{l}$ & $25340000^{l}$ & $25345000^{l}$ & $25350000^{1}$ & $25355000^{l}$ & $25360000^{l}$ & $25365000^{l}$ & $25370000^{l}$ \\
\hline & \multicolumn{9}{|c|}{ Mouse mRNA } \\
\hline ВС059910.1 & \multicolumn{9}{|c|}{ 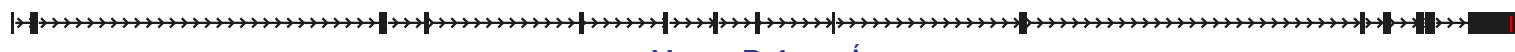 } \\
\hline $\mathrm{CpG}$ & & & & & & & 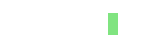 & & \\
\hline
\end{tabular}

C

\begin{tabular}{|c|c|c|c|c|c|c|c|c|c|}
\hline $\begin{array}{r}\text { Human } \\
\text { chr16 }\end{array}$ & 1 & $1360000 \mid$ & $1365000 \mid$ & $1370000 \mid$ & 1375000 & $1380000 \mid$ & $1385000 \mid$ & $1390000 \mid$ & $1395000 \mid$ \\
\hline
\end{tabular}

Fused transcript

UNKL

C16orf28

PolyA DB
Poly(A) SVM

$\operatorname{Poly}(A)$ SVM
CpG प世t

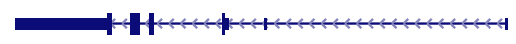

Human Refgene

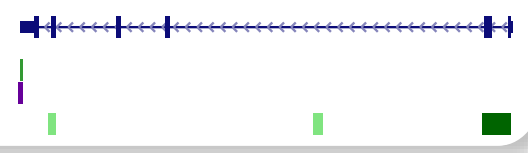

